\title{
Perspective
}

PERSPECTIVE Actualité en histoire de l'art

$2 \mid 2020$

Danser

\section{Crise, critique, mélancolie}

Crisis, Criticism, Melancholy

Krise, Kritik, Melancholie

Crisi, critica, melanconia

Crisis, crítica, melancolía

\section{Rémi Labrusse}

\section{(2) OpenEdition}

Journals

Édition électronique

URL : https://journals.openedition.org/perspective/21820

DOI : $10.4000 /$ perspective. 21820

ISSN : 2269-7721

Éditeur

Institut national d'histoire de l'art

Édition imprimée

Date de publication : 30 décembre 2020

Pagination : 261-274

ISBN : 978-2-917902-90-5

ISSN : $1777-7852$

Référence électronique

Rémi Labrusse, "Crise, critique, mélancolie », Perspective [En ligne], 2 | 2020, mis en ligne le 30 juin 2021, consulté le 30 juillet 2022. URL : http://journals.openedition.org/perspective/21820; DOI :

https://doi.org/10.4000/perspective. 21820 


\title{
Crise, critique, mélancolie
}

\author{
Rémi Labrusse
}

\begin{abstract}
- BUBENIK, 2019 : Andrea Bubenik (dir.), The Persistence of Melancholia in Arts and Culture, actes du colloque (Brisbane, 2014), New York / Londres, Routledge, 2019.

- HOLLY, 2013 : Michael Ann Holly, Melancholy Art, Princeton / Oxford, Princeton University Press, 2013.

- MERBACK, 2017 : Mitchell B. Merback, Perfection's Therapy: An Essay on Albrecht Dürer's Melencolia I, New York, Zone Books, 2017.

- VERDIER, 2020 : Aurélie Verdier, Aujourd'hui pense à moi. Francis Picabia, Ego, Image, Dijon, Les Presses du réel, 2020.
\end{abstract}

Mélancolie, aigre château des aigles ${ }^{1}$

Dans les souterrains de la conscience occidentale moderne, la mélancolie n'en finit pas de s'agiter. Elle les habite avec une violence plus ou moins aiguë selon les temps, suivant des phases successives de retrait et de recharge de sa puissance expressive, étouffée ou triomphante, renversée en fureur, célébrée en langueur. Ça bouge, ça n'avance pas : on tourne en rond. C'est comme si, depuis toujours, l'ambivalence fondamentale des pensées de la mélancolie, retournant le négatif en positif, l'aveuglement en extra-lucidité, l'impuissance en surpuissance, le mort en vivant et inversement, les constituait en un noyau d'énergie noire attirant à lui toujours plus d'indéfinissable matière. Un trou noir, en somme, autour duquel gravite et finit toujours par s'écraser tout un nuage de notions : de grands astres éteints comme l'acédie médiévale, des planètes depuis toujours poussiéreuses comme la nostalgie ou rapidement refroidies comme la neurasthénie, de petits astéroïdes incandescents comme la névrose d'angoisse ou des nébuleuses comme le spleen et de gros monstres gazeux comme la dépression - auxquels s'ajoutent à l'occasion, circulant entre les systèmes selon des trajectoires plus ou moins erratiques, la colère (une colère noire), la hantise, l'ennui, l'inspiration et le génie, le ressentiment, l'humour, la clairvoyance, la rêverie, la nausée, le nihilisme, le désespoir, le sarcasme et le blasphème, le suicide, les zombies, la grâce et même l'élégance, fille de la douleur. La mélancolie : " cette Babel ", écrivait Raymond Klibansky en 1989, où " chacun croit comprendre, ne fût-ce qu'en ses propres termes, ce que l'autre veut dire ${ }^{2}$ ». 
Pourtant, ce ne sont pas les efforts de domestication taxinomique qui ont manqué : l'acédie a été installée parmi les sept péchés capitaux et ses "filles " listées au nombre de six, sept ou soixante-huit ${ }^{3}$; la mélancolie a été isolée parmi les quatre tempéraments hippocratiques (le sanguin, le colérique, le flegmatique et le mélancolique) ou simplement opposée à la manie et elle-même divisée en trois significations distinctes quoique partiellement superposées (maladie avérée, configuration psychique stable, simple humeur passagère $)^{4}$. Dans le dédale de ces efforts qui, sous des dénominations diverses, se sont perpétués de Platon à Hippocrate, à Aristote et à ses suiveurs, aux Pères de l'Église, aux philosophes scolastiques, aux humanistes de la Renaissance, aux penseurs romantiques et aux savants positivistes du XIX ${ }^{e}$ siècle, aux médecins philosophes et aux phénoménologues de notre temps, une tension se manifeste cependant continûment, qui résulte d'une obstination à placer la mélancolie et ses avatars entre science et métaphysique - ou plutôt, de l'obstination à faire de la mélancolie le lieu même, emblématique, de la rencontre entre science et métaphysique. La spiritualité la concerne autant que la médecine ; elle se donne simultanément comme repli hors de la plénitude divine (le recessus a bono divino de Thomas d'Aquin ${ }^{5}$ ) et comme nosographie des influences réciproques du corps et de l'esprit (les humeurs). À cette insistante tension, la modernité a voulu mettre fin en la faisant basculer une fois pour toutes du côté médical, suivant un diagnostic aux tonalités décidément négatives : la notion de dépression a ambitionné de se substituer à la vieille mélancolie en ne gardant d'elle que sa face stérile et malade, pour en proposer la guérison ; la neurasthénie, effondrement du système nerveux, est allée dans le même sens, tout en se distinguant par un aspect politique et social, directement rattaché par son inventeur américain, George Beard, aux effets pervers du capitalisme industriel ${ }^{6}$, jusqu'à déboucher sur l'étiquette vaguement grotesque du burn-out, où l'antique consomption de l'âme se trouve dissoute dans des odeurs de moteur grillé.

Et malgré tout, l'ambivalence des connotations charriées par le fleuve de la mélancolie n'a jamais cessé de la maintenir, plus ou moins souterrainement, aux racines de notre sensibilité moderne. L'immense fortune du texte de Sigmund Freud, "Deuil et mélancolie ", écrit en 1915, paru en 1917², l'atteste : loin d'un strict positivisme, en situant la mélancolie au cour d'une théorie du désir, il projette autant ce dernier sur l'horizon infini de l'étrangeté à soi du sujet qu'il replie la mélancolie elle-même sur un horizon médical. La symptomatologie psychanalytique d'un désordre du désir aboutit, on le sait, à la définition d'une déviance du processus de deuil, un deuil dont le sujet s'emploie à ne pas connaître l'impossible objet, au point qu'il lui devient loisible de transformer sa perte en régression narcissique. Le moi se polarise alors indéfiniment sur une fascination éplorée à l'égard de sa propre perte - posture d'autant plus endurante qu'elle est paradoxale. Pour suggérer l'extraordinaire résistance de la constellation mélancolique à travers les époques, Giorgio Agamben a proposé de relier cette analyse freudienne aux pensées patristiques de l'acédie ${ }^{8}$, définie comme attitude contemplative poussée à l'extrême, jusqu'à la perte de contrôle de ce mouvement d'intériorisation où s'ouvrent, par conséquent, des abîmes ontologiques peuplés de fantasmes. De tels abîmes, rien n'a jamais pu conjurer l'attraction. Aucune célébration de la résilience n'en vient à bout ; au contraire, tout se passe aujourd'hui comme si c'était le sentiment acéré de leur actualité dont le rasoir mettait à nu le côté dérisoire, en regard des injonctions comportementalistes à la bonne gestion des humeurs et à la performance de soi.

Au moment où l'on croit voir la mélancolie s'éloigner à l'horizon de l'histoire et pouvoir par conséquent la circonscrire comme un objet clos offert à l'analyse rétrospective, on sent bien que sa puissance contaminante - celle du mot même et celle de ses résonances profondes - perdure. L'insistance du leitmotiv de l' « aigre château des aigles » dans l'œuvre 
de Marcel Broodthaers le dit excellemment. Simple vers d'un poème en 1947, il est l'expression d'une adhésion lyrique (quoique non dépourvue d'ironie) à la traditionnelle posture mélancolique de l'écrivain. Puis se produit une rupture cynique, en 1964, par laquelle celui-ci renonce au lyrisme littéraire pour la rentabilité des objets d'art : " Moi aussi, je me suis demandé si je ne pouvais pas vendre quelque chose et réussir dans la vie. Cela fait déjà un moment que je ne suis bon à rien. Je suis âgé de quarante ans. L'idée, enfin, d'inventer quelque chose d'insincère me traversa l'esprit et je me mis aussitôt au travail ${ }^{9}$. " Mais la mélancolie fait aussitôt retour dans cette nouvelle entreprise comme une hantise irriguant un processus infini

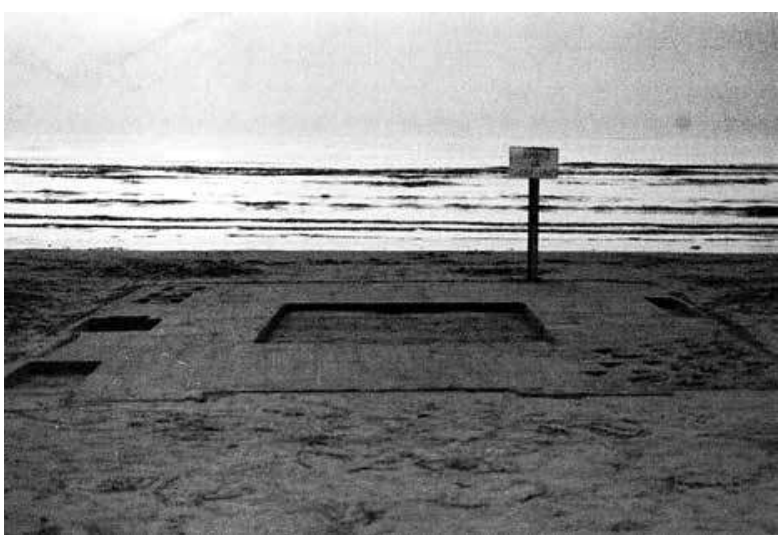

1. Marcel Broodthaers, Musée d'art moderne, département des aigles, section documentaire, Le Coq (Knokke-le-Zoute), été 1970, photographie de Maria Gilissen. d'autocritique : le "musée " parodique créé par l'artiste entre 1968 et 1972 prend le nom de « Département des aigles " (fig. 1), nom "né d'un poème, un très vieux poème " qui, précise-t-il, lui donne le statut de "souvenir littéraire ${ }^{10}$ ». Or, précisément parce que le propre de l'horizon mélancolique est de constituer le présent en état paradoxal de toujoursdéjà-passé, ce statut de souvenir, loin de mettre à distance la posture mélancolique, en revivifie plutôt la puissance présente de dérangement, jusque dans l'objet dans lequel elle se fige lorsque « l'aigre château des aigles " réapparaît en gravure sur une plaque de bronze.

Persistance de la mélancolie : c'est le titre du volume collectif dirigé par Andrea Bubenik, The Persistence of Melancholia in Arts and Culture, à partir d'un colloque organisé en Australie à l'occasion du 500 anniversaire de la gravure allégorique de Dürer, Melencolia I (fig. 2) . Appuyées essentiellement sur cette œuvre et sur l'article de Freud, les onze contributions éclairent le puits sans fond de significations dont l'image est porteuse et son " pouvoir quasi contraignant sur l'imagination de la postérité ${ }^{11}$ ", comme l'avaient déjà formulé en leur temps Raymond Klibansky, Erwin Panofsky et Fritz Saxl, jusqu'à la modernité à laquelle s'attachent plus particulièrement les auteurs du volume. Melencolia I y apparaît plus que jamais comme un Denkbild au sens de Benjamin"12, un " objet théorique " au sens $\mathrm{d}^{\prime}$ Hubert Damisch ${ }^{13}$, enraciné dans l'imaginaire occidental pour y mettre en mouvement la pensée, qu'il s'agisse de littérature (de Victor Hugo à Winfried G. Sebald), d'arts visuels ( $\mathrm{d}$ 'Odilon Redon à Alberto Giacometti et à Anselm Kiefer), de cinéma (de Stanley Kubrick - avec le monolithe de 2001, l'Odyssée de l'espace, écho du polyèdre énigmatique de la gravure - à Lars von Trier - avec son Melancholia de 2011) ou de musique (évoquée dans la contribution de Denis Collins à travers les compositeurs Johann Nepomuk David, Harrison Birtwistle et James Wilding $\left.{ }^{14}\right)$. Le temps n'est plus, manifestement, aux tentatives de décryptage de la signification cachée de l'œuvre de Dürer, mais à l'observation et au développement de sa capacité à tisser des liens entre le passé et le présent, aussi bien qu'entre les cultures : ainsi Drew Daniel s'appuie-t-il librement sur le désordre des objets accumulés et délaissés au pied de l'allégorie ailée de 1514 pour reconsidérer le phénomène actuel de rejet du monde désigné en japonais sous le nom d'hikikomori, inversion de la vis contemplativa monastique dans l'univers du nihilisme consumériste, traversée par la terreur obsessionnelle d'une vacuité ontologique qu'emblématise la prolifération des objets dans l'univers clos d'une chambre-cellule ${ }^{15}$ (fig. 3). 


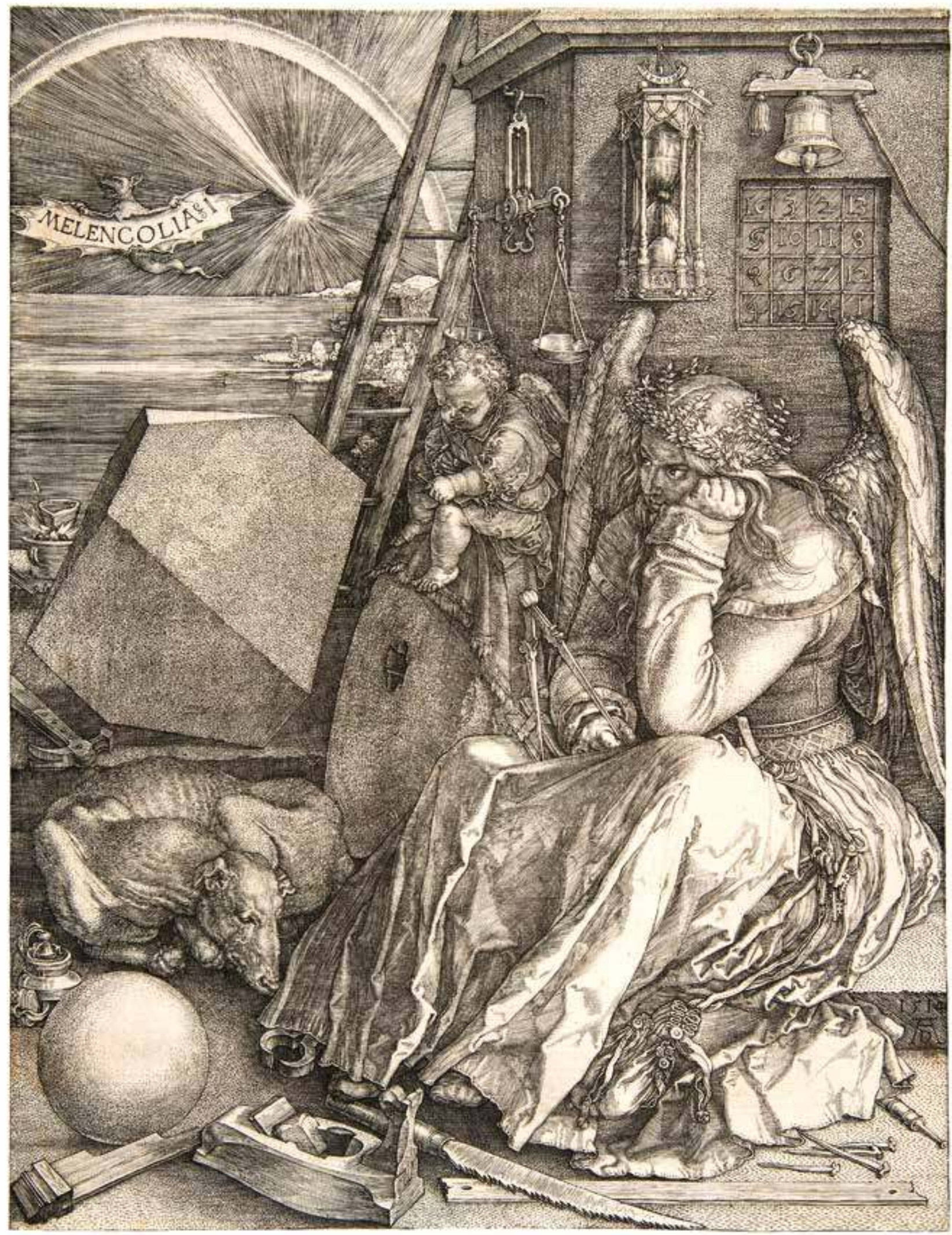


De fait, si Melencolia I s'est si profondément installée dans nos représentations, bien au-delà de la sphère savante, c'est précisément par sa résistance au décryptage qui en ferait une structure sémantique close. Le sentiment de sa contemporanéité, et donc sa productivité continue, naissent de l'impossibilité d'insérer ses composants iconographiques dans une narration univoque que leur netteté hallucinatoire ne peut pourtant pas ne pas appeler à l'esprit. Ainsi la gravure magistrale (le Meisterstich, comme on l'appelait dans le milieu de Dürer) répond-elle anachroniquement à la définition freudienne de la mélancolie en tant qu'exacerbation du désir pour un objet que le sujet s'est lui-même employé à rendre inaccessible. Son emblème, suggère Mieke $\mathrm{Bal}^{16}$, est le polyèdre sur une face-miroir duquel une tache fantomatique pourrait représenter le reflet monstrueux du visage et du poing fermé de l'ange allégorique : objet inappropriable que Giacometti a rebaptisé Cube ou Pavillon nocturne (fig. 4) en y gravant, lui, un autoportrait ${ }^{17}$, et dont l'indétermination sémantique exprime emblématiquement l'angoisse de "la forme des choses à venir ", écrit Andrea Bubenik, paraphrasant Herbert G. Wells ${ }^{18}$.

L'insistance contemporaine sur les significations flottantes de la plus célèbre gravure de l'humanisme européen oblige ici à revenir un moment sur les grands axes du débat qui en structure l'analyse, depuis plus d'un siècle. Philippe Despoix ${ }^{19}$ a récemment repris l'histoire de cette analyse qui commence en 1903 avec la première étude de Carl Giehlow sur le cercle humaniste que fréquentait Dürer autour de l'empereur Maximilien I I 20, et qui se poursuit jusqu'en 2019 avec la réédition anglaise mise à jour de l'opus magnum de Klibansky, Panofsky et Saxl, Saturne et la mélancolie ${ }^{21}$. La première publication de ce texte s'étend elle-même de 1923, lorsque Saxl et Panofsky font paraître une longue analyse de Melencolia I dans les Studien der Bibliothek Warburg22, jusqu'à 1964, date de l'édition originale en anglais du livre devenu, après bien des péripéties, une histoire générale de l'idée de mélancolie en Occident depuis l'Antiquité, grâce notamment à l'intervention de Klibansky. Mais le livre doit aussi énormément à Aby Warburg, non seulement parce qu'il a été principalement écrit dans le cadre de la bibliothèque Warburg, et grâce à ses ressources, mais aussi et surtout parce qu'il a été précédé par les études de Warburg lui-même, autour de 1913, sur le rôle de la divination antique et des représentations astrales dans la pensée humaniste dont la gravure de Dürer constitue une expression majeure ${ }^{23}$.

Si, un siècle après le début de sa conception, Saturne et la mélancolie se présente toujours comme un des grands sommets de l'histoire des arts et des idées, si aucune étude récente sur Dürer ou sur l'idée de mélancolie ne manque de le citer ni de se positionner par rapport à lui, c'est certainement du fait de la monumentale érudition dont ce texte fait preuve et, plus spécifiquement, de l'exposé paradigmatique qu'il contient de la méthode iconologique panofskyenne appliquée à la gravure de Dürer; mais c'est aussi parce que, autour de l'interprétation de cette œuvre, a pris corps un débat dont les deux protagonistes sont Warburg et Panofsky. La relation de ce dernier à son mentor, à la fois filiale et brutalement infidèle, aboutit à la fois

2. Albrecht Dürer, Melencolia I, 1514, estampe, $24,1 \times 18,5 \mathrm{~cm}$, New York, The Metropolitan Museum of Art.

3. Tatsuhiro Satō, le personnage hikikomori de la série animée japonaise Bienvenue dans la NHK (NHK にようこそ!, NHK ni yōkoso!, Gonzo, 2006), d'après le roman original de Tatsuhiko Takimoto illustré par Yoshitoshi Abe (Tōkyō, Kadokawa Shoten, 2002).

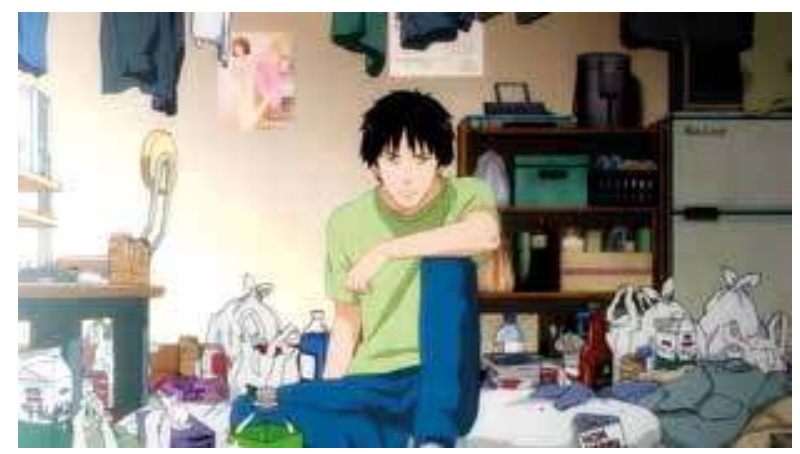




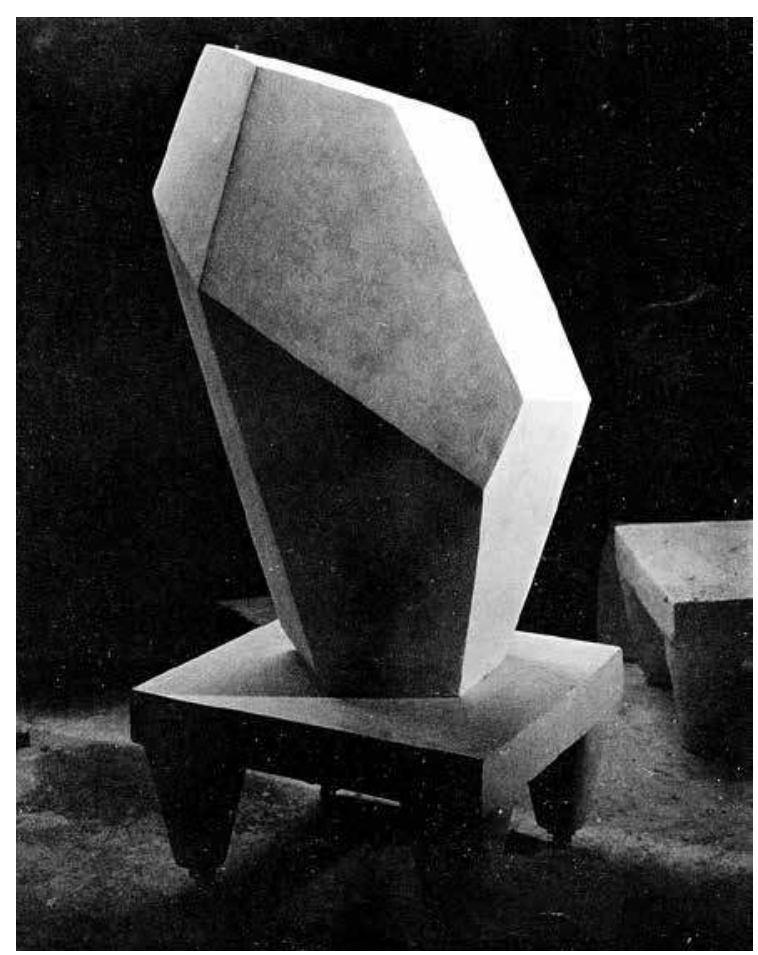

4. Alberto Giacometti, Le Cube (Pavillon nocturne), 1933-1934, plâtre, photographie reproduite dans Minotaure, $\mathrm{n}^{\circ} 5,1934$, n. p. à la reprise des éléments mis au jour par Giehlow et Warburg - en particulier l'influence probable sur Dürer du manuscrit De occulta philosophia (1510) de l'humaniste ésotériste Agrippa von Nettesheim - et au renversement de l'interprétation qui en découle. Pour Warburg, se fondant sur le carré magique accroché au mur derrière la figure de l'ange, la gravure est une image apotropaïque ou, suivant sa formule célèbre, un Trostblatt, une " feuille de réconfort humaniste contre la crainte qu'inspire Saturne ${ }^{24} "$ : la primauté serait ainsi accordée à la raison pour conjurer les forces obscures de l'inconscient. Pour Panofsky, au contraire, tous les éléments iconographiques de l'image concourent à en faire la représentation catastrophique d'une perte de foi dans les pouvoirs accordés par l'humanisme à l'art et à la pensée ; à ce premier stade de la mélancolie - celui de la melencolia imaginativa -, l'individu prendrait " une sombre conscience de l'insuffisance de ses pouvoirs de connaître, car il manque à son esprit la capacité de donner aux facultés supérieures leur libre effet $^{25}$ " (ce qui, en revanche, se produit au troisième et dernier stade de l'inspiration mélancolique, celui de la melencolia contemplativa).

Ce débat ne se limite pas à la question du sens des motifs allégoriques empruntés par Dürer à la philosophie de la Renaissance. Parallèlement se joue une controverse sur les méthodes et le sens de l'histoire de l'art : ou bien, comme chez Panofsky, celle-ci tend à postuler l'existence d'une signification spécifique, enracinée dans son temps, que l'enquête iconologique de l'historien d'art a pour tâche de remettre au jour en séparant clairement le passé du présent ; ou bien, comme chez Warburg, l'intuition d'une migration perpétuelle des motifs et de leurs significations aboutit à mettre l'accent sur l'action des images à travers le temps, au-delà de leurs intentions constitutives, c'est-à-dire à privilégier une pensée de leur productivité par rapport à une pensée de leur essence. Comprendre Melencolia I, est-ce découvrir la clé d'une énigme ou adhérer à un flux d'énergie imaginative, à un " espace de pensée " (Denkraum) ?

Ce débat demeure ouvert aujourd'hui : par exemple, tandis que Christopher Wood, à l'occasion de la réédition en anglais de Saturne et la mélancolie, a pris fait et cause pour la lecture panofskyenne, accusant Warburg d'avoir « fermé les yeux sur le contenu tragique de la gravure ${ }^{26}$ ", Mitchell B. Merback, dans un livre remarqué, a au contraire ravivé l'actualité de l'interprétation warburgienne de Melencolia I en tant que Trostblatt. Pour lui, l'image constitue non seulement un diagnostic de l'affection mélancolique mais aussi un remède effectif, inscrit au registre des artefacts thérapeutiques : si elle prend l'allure d'une énigme, ce serait précisément pour décevoir tous les efforts d'interprétation et aider le spectateur-exégète - c'est-à-dire l'artiste lui-même, son cercle d'amis et, in fine, toute la communauté des croyants - à aller au-delà de la prétention humaniste à parvenir à une élucidation parfaite du monde par le langage et par les images (considérées comme 
une forme d'écriture). Melencolia I, image " parfaitement imparfaite ${ }^{27}$ ", contrebalancerait ainsi l'hybris de l'intellect humaniste par le recours à une thérapie " astro-médico-philosophique $^{28}$ » de nature homéopathique et d'inspiration intensément chrétienne. L'enquête iconographique - mue par le fantasme d'un hypothétique " Dürer Code " - cède alors le pas à une réflexion sur les formes de pensée : en l'occurrence celle du pouvoir médical accordé aux images. À l'image comme représentation se substitue l'image comme dispositif d'action sur le corps et l'esprit, via le regard.

Ce qui, en fin de compte, ressort de cette enquête sur la nature de l'agentivité figurative, en Allemagne, au début du XVI ${ }^{e}$ siècle, c'est une liberté accrue des mises en rapport à travers les époques, recourant aussi bien à Pétrarque qu'à Freud voire, à l'occasion, au bouddhisme et à John Cage. Et ce qu'on doit constater une fois de plus, ce faisant, c'est l'extraordinaire adaptabilité de la grande et mystérieuse image de Dürer à l'évolution des sensibilités savantes : en l'occurrence, la voici réceptrice, sur un plan méthodologique, de la fascination contemporaine pour la démarche warburgienne et de la prise en compte des vertus herméneutiques de l'anachronisme ; sur un plan esthétique, de la sensibilité à une poétique moderniste, au sens large, de l'imperfection positive ( "l'imperfection est la cime ", comme dit un poème d'Yves Bonnefoy) ; sur un plan culturel, enfin, de la conception poststructuraliste de la pensée comme un exercice infini d'agencement des significations. S'y ajoute l'intégration de cette conception non pas à un scepticisme dit " postmoderne " mais à un intérêt pragmatique pour

5. Albrecht Dürer, Traumgesicht [« in der Nacht im Schlaff hab ich dis Gesicht gesehen, wy fill großer Wassern vom Himmell fillen »], 1525, plume et aquarelle sur papier, $30 \times 43 \mathrm{~cm}$, Vienne, Kunsthistorisches Museum.

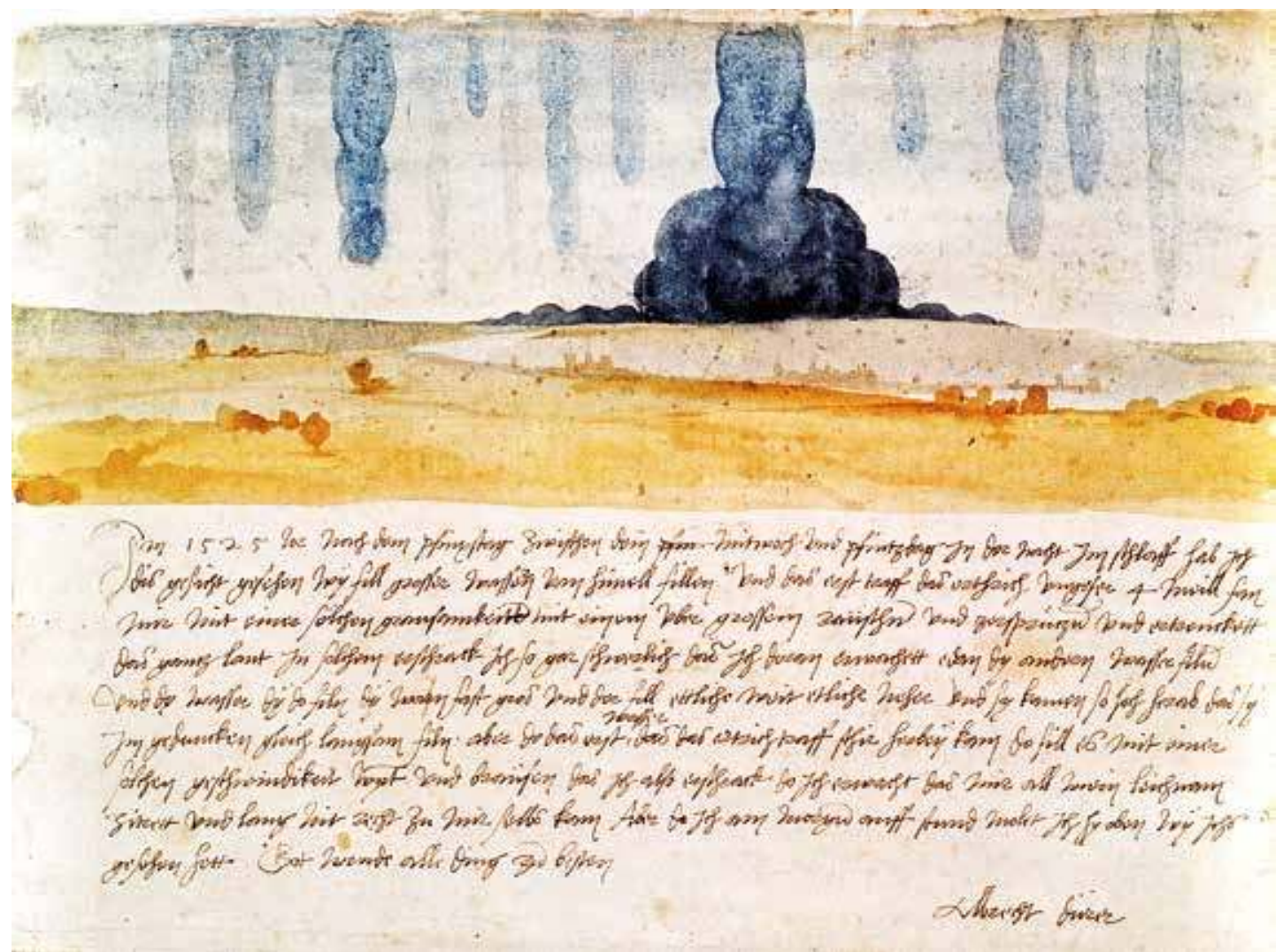


tout ce qui ressortit aux thérapies parallèles et aux multiples stratégies du soin, dans un temps d'aggravation des incertitudes existentielles et des angoisses apocalyptiques. De là vient l'admiration particulière suscitée, de nos jours, par la feuille tardive où Dürer, trois ans avant sa mort, dans un extraordinaire acte d'auto-analyse, a consigné l'un de ses cauchemars sous forme de déluge pré-atomique balayant le monde (fig. 5).

En somme, ce qui confère son insistante actualité à Melencolia I, c'est d'avoir associé en une seule image la spéculation critique et le pathos de la crise ; autrement dit la " vigilante attention à soi $^{29}$ " et la douleur d'un état de séparation d'avec soi, que le seul fait de sa formulation rend inguérissable. Crise et critique, de fait, se rejoignent dans la mélancolie ; dans la mesure où cette union caractérise le destin occidental moderne, sous l'empire de l'esprit scientifique, on peut comprendre que la mélancolie en soit devenue l'affect fondamental, auquel Dürer donne sa profondeur temporelle maximale, manifestant l'unité de son déploiement de la Renaissance à nos jours. Le régime de la conscience critique est celui du clivage : il découpe le monde en objets et en sépare le sujet observant, lequel en vient à devoir se considérer lui-même en tant qu'objet. Le déracinement, le complexe d'irréalité, la douleur, en un mot, qui en résultent font de la découpe une schize et de la conscience critique une crise ${ }^{30}$. L'esprit scientifique, en d'autres termes, c'est-à-dire l'esprit critique, produit affectivement, au cœur de la culture moderne, un état de crise, avec tout ce que cette expression comporte d'oxymorique : une crise, en effet, c'est un mouvement, un pli, un nœud emporté vers son dénouement - et voici que ce mouvement, dans son instabilité même, se perpétue désormais en état sans résolution. Cet état de crise propre à la culture critique de l'Occident moderne est à la crise comme événement ce que, dans le vocabulaire freudien, la mélancolie est au deuil : tandis que, dans le deuil, la perte de l'objet aimé se résout en transfert vers un nouvel objet, cette perte s'intériorise dans la mélancolie en contemplation de soi ; de même, tandis que l'épreuve classique de la crise engendre l'émergence d'un nouvel ordre, la crise propre au criticisme moderne devient en tant que telle un mode d'être paradoxal, au sein duquel les alternances de la manie et de la mélancolie, de l'hybris et de l'inaction se suivent indéfiniment et s'aggravent sans se dénouer.

Parmi les constituants fondamentaux de cet état de crise - inséparablement culturel et individuel - figure au premier plan l'emmêlement des dimensions du temps. "The time is out of joint ", le temps est hors de ses gonds, désarticulé, " désajointé ", annonce Hamlet - figure de la mélancolie au moins aussi puissante dans nos imaginaires que l'ange de Dürer -, après avoir parlé avec le spectre de son père assassiné. Ce trouble dans la temporalité que l'image de Saturne, alias Kronos, a associé à la mélancolie constitue aujourd'hui encore un point focal vers lequel convergent toutes les analyses, en s'appuyant avec prédilection sur les travaux de la psychanalyse, de Freud à Melanie Klein ${ }^{31}$ et à Donald W. Winnicott, son disciple, selon lequel la posture mélancolique se manifeste par « la recherche mémorielle infinie de quelque chose dans le futur qui réside dans le passé32 ".

La sensibilité à la crise du vécu temporel, qui serait le propre de la mélancolie, est centrale, notamment, dans l'ouvrage de Michael Ann Holly, Melancholy Art, où l'auteure, sur le ton de la confidence érudite, ancrée dans des épreuves personnelles traumatiques, part d'une méditation freudienne sur la «blessure ouverte ${ }^{33}$ " entre le passé et le présent que les œuvres d'art ancien rendent manifeste, pour faire l'hypothèse que l'histoire de l'art, dans son ensemble, est par nature une discipline mélancolique, un mouvement de pensée obsédé par l'expérience du manque et de l'absence, quand bien même on voudrait l'étouffer par les mille et un stratagèmes du positivisme documentaire et de l'attributionnisme ${ }^{34}$. L'objet de l'histoire de l'art est irrémédiablement absent : parce que la représentation ne donne pas la présence et parce que l'abîme ne se comble jamais entre le visuel et le verbal, ni entre 
le passé et le présent. Autrement dit, la contemporanéité matérielle et affective des œuvres avec leurs regardeurs tardifs révèle - plus intensément que dans les autres disciplines historiques - le manque de synchronicité entre eux et exacerbe le sentiment d'une "perte en monde " qui ne peut pas ne pas en découler. Mais cette perte, à son tour, produit une forme singulière d'expérience et de savoir que seule peut rejoindre, selon Michael Ann Holly, une poétique de l'histoire de l'art, loin du puritanisme savant arc-bouté, quant à lui, sur le refoulement des affects, face à l'énigme de " présences matérielles excédant toute compréhension ${ }^{35}$ ". Des figures inspiratrices contribuent à donner corps à cette poétique, parmi lesquelles Michael Baxandall, rejoint dans les couches profondes de sa sensibilité, en amont de son discours professoral, ou Adrian Stokes, écrivain et chercheur mais aussi peintre et psychanalyste, patient de Melanie Klein, dans ses Stones of Rimini de 1934. Une fois de plus, Aby Warburg est également mobilisé à de nombreuses reprises, ainsi que, dans son sillage, Georges Didi-Huberman, non sans que soit brouillé, ce faisant, l'écart pourtant frappant entre la "poétique de la perte " selon Michael Ann Holly ${ }^{36}$ et la foi - fût-elle utopique - de Warburg et de ses admirateurs dans les capacités de l'anachronisme à engendrer un "gai savoir " aux antipodes de la mélancolie ${ }^{37}$.

Au nombre des références sur lesquelles s'appuient en général ces réflexions récentes de l'histoire de l'art sur la mélancolie, de Freud à Klein, de Walter Benjamin à Giorgio Agamben, de Jacques Derrida à Julia Kristeva, un auteur manque singulièrement ou n'est évoqué que fugitivement : c'est Ludwig Binswanger, qui fut pourtant le psychiatre de Warburg à la clinique Bellevue de Kreuzlingen entre 1921 et 1924, qui assista par conséquent à la conférence de ce dernier sur le rituel du Serpent chez les Indiens Hopi, le 21 avril 1923, et qui continua à correspondre avec son ex-patient après sa sortie de la clinique, lorsque Warburg se consacra entièrement à la constitution de son atlas d'images Mnémosyne. "Cette manière de tracer un arc de cercle en arrière [...] représente aussi une tension vers l'avant ", lui écrit par exemple Binswanger ${ }^{38}$, signalant par là l'importance de la notion warburgienne de survivance dans l'élaboration de sa propre démarche, entre psychanalyse et phénoménologie, pour analyser la désorganisation des structures temporelles dans le complexe mélancolique. Pour Binswanger ${ }^{39}$, en effet, si la mélancolie donne paradoxalement accès au réel à travers le prisme de sa perte, c'est parce qu'elle résulte d'un trouble dans la constitution de la temporalité, au niveau transcendantal : le mouvement psychique de rétention (c'est-à-dire la constitution du passé) contamine et altère les mouvements de présentation et de protention vers un futur qui se trouve donc toujours déjà hypothéqué par le regret. La rétention est vécue sur le mode de la protention, tandis que la protention est vécue, elle, sur le mode de la rétention. Dans cet enchevêtrement paralysant de l'intentionnalité, le présent n'est plus ni soutenu, ni mis en mouvement, et sa qualité de présence s'étiole irrémédiablement. À l'intérieur de ce schéma, on peut penser que l'itinéraire de Warburg (lequel analysait lui-même son Rituel du Serpent comme " la confession d'un schizoïde [incurable] $]^{40}$ ") a incarné aux yeux du psychiatre-phénoménologue la victoire improbable, quasi miraculeuse, grâce à la relation aux ouvres d'art, de la puissance créatrice sur la fossilisation psychique dans la pathologie mélancolique ; la victoire, autrement dit, de la melancholia generosissima, attribuée à Dürer par Melanchthon ${ }^{41}$, sur la "schizophrénie " ou "l'état mixte maniacodépressif " diagnostiqué par la science ${ }^{42}$.

Qui dit temps désordonné dit résurgence des fantômes. Tandis que s'écroulent les fondations mêmes de sa présence au monde, le moi dépérit et recherche son reflet ruiné dans les objets qu'il fait proliférer en les dotant à leur tour d'une pseudo-vie crépusculaire : des spectres. À la mélancolie se rattache donc l' « hantologie », cette science à laquelle Jacques 
Derrida, en l'inventant, a assigné la mission d'explorer le domaine des êtres qui circulent indistinctement entre objet, image et vivant ${ }^{43}$. Le capitalisme, père de la neurasthénie, a constitué un terreau privilégié pour la démultiplication de ces spectres auxquels nous lient désormais les diverses formes du fétichisme consumériste et qui, parallèlement et sans surprise, nourrissent l'inclination croissante de l'histoire de l'art pour des fantasmes animistes. Mélancolique, l'histoire de l'art ne l'est sans doute pas tant par sa relation générique au passé que, d'abord, par un partage des spectres dont elle est redevable au matérialisme capitaliste. C'est dans son cadre, et sous ses injonctions, qu'elle a connu le développement démesuré qu'on lui connaît aujourd'hui, notamment dans les musées ; c'est lui qui l'a instituée en instance de sublimation quasi sacrale de son propre objectivisme - de ses spectres. Vouloir transmuer l'obsession des objets en culte des objets, recouvrir (donc couronner et cacher à la fois) les produits de la techno-industrie par les idoles de l'art, comme on l'assigne aux divers dispositifs de l'histoire de l'art, c'est vouloir conjurer la puissance mélancolique à l'œuvre dans la culture industrielle moderne, mais c'est aussi, par là même, en reconnaître l'action contaminante. À nouveau, crise et critique ne font qu'un, et de leur insupportable enlacement naît le désir de s'en dégager par l'hypothèse de l'animisme. Quelle que soit la distance entre leurs conceptions de l'histoire de l'art - mélancolie d'un côté, gai savoir de l'autre -, c'est ce qui rapproche Michael Ann Holly, lorsqu'elle suggère que " les vies futures des objets sont aussi riches et denses que la nôtre ${ }^{44}$ " et Georges Didi-Huberman, lorsqu'il admire la capacité de Warburg à « voir une âme dans chaque chose, si modeste fût-elle ${ }^{45} "$. Cet animisme n'en reste pas moins sur le registre du " comme $\mathrm{si}^{46} "$, de l'aveu même de ses célébrants, et finit donc à son tour par renvoyer au vide

6. Giorgio de Chirico, Mélancolie hermétique, décembre 1918-janvier 1919, huile sur toile, $62 \times 50 \mathrm{~cm}$, Paris, musée d'Art moderne de la Ville de Paris.

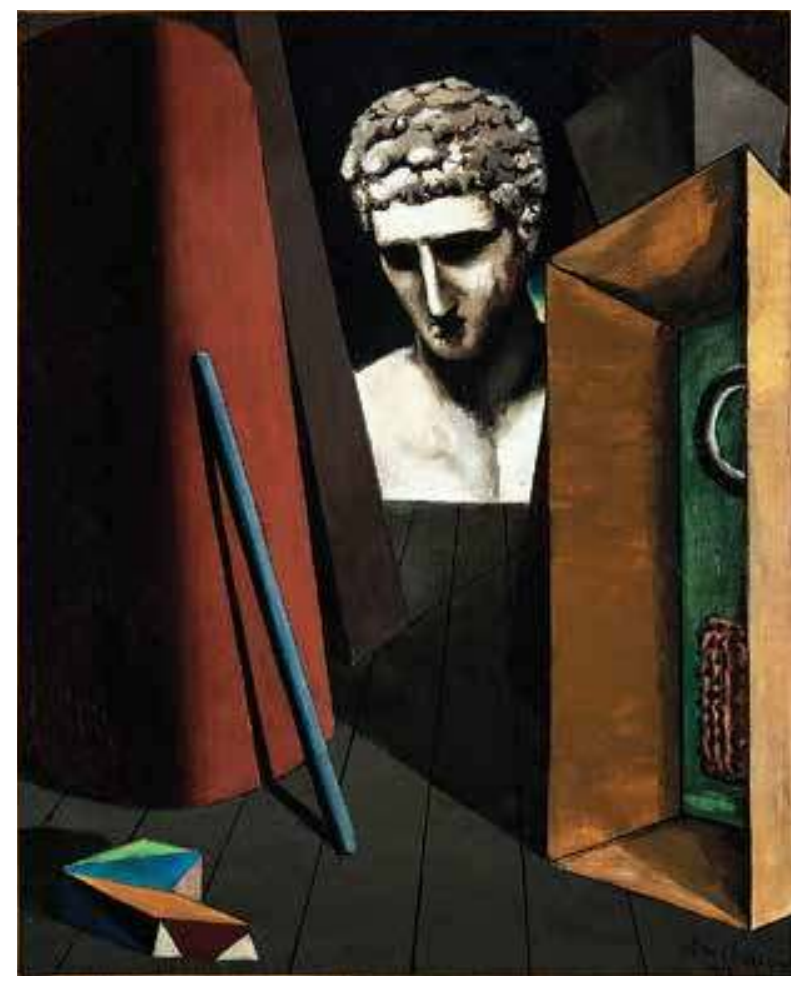
qui l'a engendré et qu'il n'a fait que refouler. Encore et toujours, la conscience critique referme sur elle-même la boucle de la crise : les objets-spectres désignent avant tout l'impossibilité de la présence réelle, tandis que les inclinations animistes, minées par l'esprit critique, se résolvent en mauvaise foi, recyclant tant bien que mal d'anciens fantasmes primitivistes.

Aux antipodes, cependant, de toute velléité animiste, nombreux sont les artistes du $\mathrm{XX}^{\mathrm{e}}$ siècle qui ont assumé une posture critique radicale en identifiant leur création - ou, disons, leur situation d'artistes - à une critique de la création, au risque de ne plus laisser subsister d'eux, volontairement, qu'une identité vide et sans œuvre. De même que le moi mélancolique s'hypertrophie paradoxalement dans la déploration de sa propre perte, cette radicalité critique construit l'hyper-lucidité de l'artiste sur un état de crise psychique et donc d'obscurcissement. Comme des enfants siamois, déconstruction et effondrement frayent monstrueusement et se menacent réciproquement. 
L'hypothèse d'Aurélie Verdier, dans $A u$ jourd'hui pense à moi. Francis Picabia, Ego, Image, ouvrage issu de sa thèse, est que Picabia fut, avant Chirico (fig. 6), Warhol, Broodthaers ou tant d'autres, et plus brutalement que Duchamp, le maître pionnier de cette posture qui renverse la vieille condamnation morale de l'acédie comme " ennui de bien faire ${ }^{47}$ " en principe constitutif, et lui confère donc une valeur spéculative. "Paresse et ennui de bien faire " : la critique de la peinture, chez Picabia, est identiquement une mélancolie de la peinture et, en elle, d'une raison d'être au monde, devenue indéterminable. D'un côté, se déploie un autodiagnostic déconstructeur du moi créateur, auquel sont substitués des spectres : pseudo-objets industriels des portraits mécanomorphes de la fin des années 1910 ; anti-icônes des taches d'encre intitulées Sainte Vierge en 1920 ; nonsignatures du " quasi-nom ${ }^{48}$ » de Picabia, seul inscrit comme un logo sur la toile ou la feuille de papier ; toiles " insincères ${ }^{49}$ " plagiées de l'impressionnisme, de cartes postales ou de magazines érotiques, plus tardivement. D'un autre côté (mais inséparablement), s'approfondit une condition pathologique que l'artiste nomme lui-même " neurasthénie » et qui le conduit à des comportements " réactionnels "

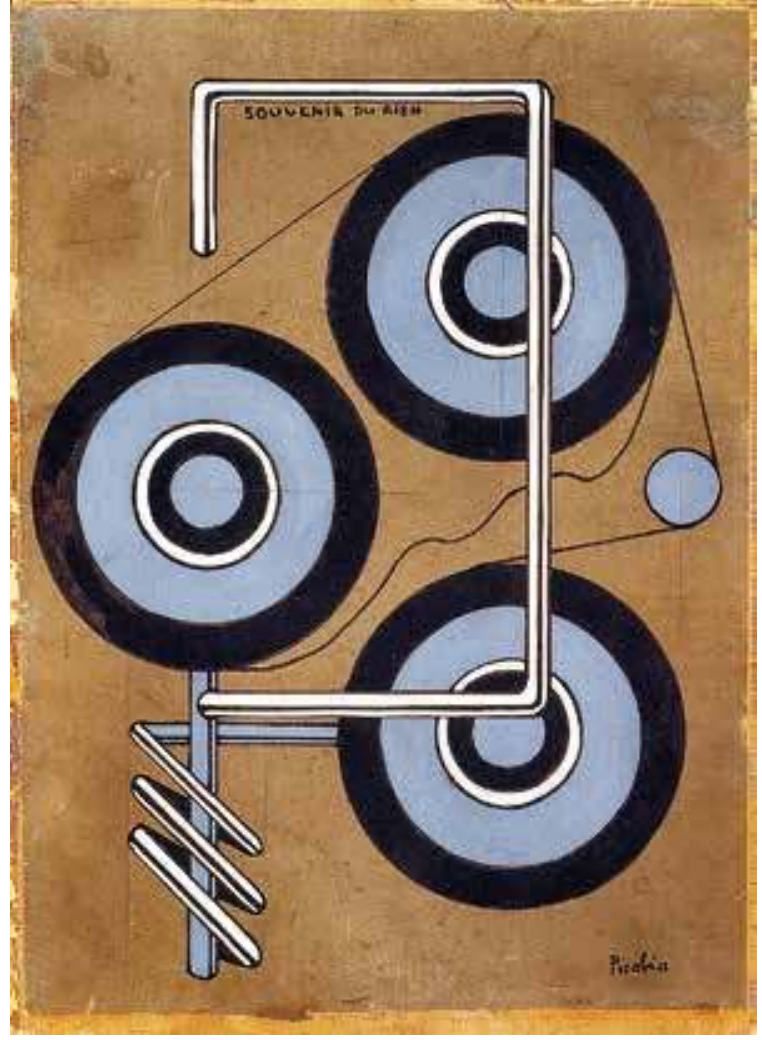

7. Francis Picabia, Souvenir du rien, vers 1919-1920 [ou 1918-1922 ?], huile et crayon sur papier marouflé sur masonite, $53 \times 38,8 \mathrm{~cm}$, Houston, The Menil Collection. dont la face politique la plus sinistre fut celle, dès les années 1920, de prises de position réactionnaires puis antisémites et pétainistes.

Selon Aurélie Verdier, " un seul sujet maniaque et mélancolique ${ }^{50}$ " est à l'origine de cette agitation qui est l'envers noir de la création, et dont le principe originel est formulé par l'artiste en 1921 sur un mode simultanément lucide et éploré : "Or, moi, je l'ai écrit bien souvent, je ne suis rien, je suis Francis Picabia ${ }^{51}$. " Comme l'indique le titre de l'ouvrage, emprunté à un poème de Picabia, l'implosion des structures temporelles est à la racine de cette dé-substantiation : la phrase "Aujourd'hui pense à moi ", en 1917, apparaît comme la cellule-mère d'une " conception de la vie et de l'art " qui est " celle d'un présent perpétuel, d'un moi sans mémoire et, peut-être, sans projection ${ }^{52}$ " et qui justifie de voir une sorte paradoxale d'autoportrait dans la toile mécanomorphe intitulée Souvenir du rien (fig. 7), deux ans plus tard.

Par ce portrait de Francis Picabia en mélancolique, Aurélie Verdier ne propose pas seulement d'appliquer une grille d'interprétation unitaire à tout le parcours de l'artiste, à contre-courant du cliché d'un génie abstrait puis dada, dans les années 1910-1920, qui aurait basculé plus tard dans le cynisme et le ressentiment. Elle dresse plus largement une allégorie de la condition mélancolique moderne, qui nous est commune, caractérisée par le double dysfonctionnement de notre relation à l'objet et de notre inscription dans le temps, sous l'ombre de la révolution techno-industrielle et du fétichisme - ou de la tyrannie - de la marchandise. 
Au total, devant l'immense bibliothèque de la mélancolie, corpus tentaculaire plus fécond que jamais, dont les innombrables textes consacrés à Melencolia I de Dürer constituent comme un microcosme, la mélancolie nous saisit : tant de livres gravitant autour de la gravitation autour du néant - le vide. On tourne en rond. Et pourtant, ce n'est pas faute de vouloir en finir, aujourd'hui plus que jamais : soit par la radicalisation antihumaniste de la mélancolie, sous les espèces de "l'estrangement sinistre ${ }^{53}$ "-l'eerie décrit par Andrea Bubenik, après Mark Fisher et Rex Butler, comme une façon de "dépasser la condition humaniste archaïque " de la mélancolie ${ }^{54}$ - ; soit au contraire par son rejet activiste, en " résistant à la mélancolie de gauche ", comme le demandait Wendy Brown en 199955, en faveur d'un ré-enclenchement de l'esprit visionnaire et du désir de transformation politique, ou en dénonçant, comme Paul Gilroy en 2004, la perpétuation de la violence propre à la "mélancolie postcoloniale ${ }^{56}$ ", cette fois en faveur d'un multiculturalisme organique, indiscipliné, buissonnant dans les grands centres urbains d'aujourd'hui.

Dans ces deux directions opposées - une ultime désactivation du rapport au monde de l'individu, d'un côté ; une revitalisation activiste du tissu social, de l'autre -, les propositions artistiques contemporaines abondent. Ensemble, elles manifestent autant l'aggravation d'une ambivalence maniaco-dépressive que l'exigence d'introduire un nouveau pli dans le désordre de l'histoire. Sans qu'on puisse trancher, la lumière d'apocalypse dont on se plaît à éclairer le présent apparaît à la fois comme l'accomplissement d'un fantasme mélancolique hissé à l'échelle planétaire et comme le début du basculement dans l'inconnu de cet affect pluriséculaire dont l'efficacité ne serait plus assurée, comme avant, par le désir obscur de sa perpétuation. 


\section{Rémi Labrusse}

Rémi Labrusse enseigne l'histoire de l'art à l'université Paris Nanterre. Il a récemment publié Face au chaos. Pensées de l'ornement à l'âge de l'industrie (Dijon, Les Presses du réel, 2018) et Préhistoire. L'envers du temps (Vanves, Hazan, 2019).

\section{NOTES}

1. Marcel Broodthaers, "Le Zodiaque » [1947], dans La Bête noire, Bruxelles, 1961.

2. Raymond Klibansky, "Avant-propos » [1989], dans Raymond Klibansky, Erwin Panofsky et Fritz Saxl, Saturne et la mélancolie. Études historiques et philosophiques : nature, religion, médecine et art (1964), Paris, Gallimard, 1989, p. 9

3. Voir Giorgio Agamben, Stanze. Parole et fantasme dans la culture occidentale (1977), Paris, Payot \& Rivages, 1981, p. 30, citant Grégoire le Grand, Isidore de Séville et, pour une liste de 68 avatars, Aurora de Michel Leiris (1946).

4. Voir Klibansky, Panofsky et Saxl, 1989, cité n. 2, p. 29.

5. Voir Agamben, (1977) 1981, cité n. 3, p. 25-26.

6. George M. Beard, La Neurasthénie sexuelle. Hygiène, causes, symptômes et traitement (1895), Paris et Montréal, L'Harmattan, 1999 [éd. orig. angl. : 1890].

7. Sigmund Freud, "Deuil et mélancolie », Métapsychologie, Philippe Koeppel (trad.), Paris, Flammarion, 2012 [éd. orig. : "Trauer und Melancholie», Zeitschrift für Psychoanalyse, Band IV, 1916].

8. Agamben, 1981, cité n. 3, p. 46-51.

9. Carton d'invitation, Bruxelles, galerie Saint-Laurent, 10-25 avril 1964, repr. dans Catherine David et Véronique Dabin (dir.), Marcel Broodthaers, cat. exp. (Paris, Galerie nationale du Jeu de Paume, 1991), Paris, Réunion des musées nationaux, 1991, p. 56.

10. Ludo Bekkers, « Gesprek met Marcel Broodthaers » [13 décembre 1969], dans Museumjournaal, 15 février 1970, p. 66-71.

11. Klibansky, Panofsky et Saxl, 1989, cité n. 2, p. 585.

12. Voir Peter-Klaus Schuster, Melencolia I : Dürers Denkbild, Berlin, Mann, 1991.

13. Yve-Alain Bois, Denis Hollier, Rosalind Krauss, « $A$ Conversation with Hubert Damisch ", dans October, $n^{\circ} 85$, 1998, p. 8, cité par Mieke Bal, « Temporal Turbulence. In Praise of Anachronism », dans BUBENIK, 2019, p. 144.

14. Denis Collins, "Dürer, Music, and Melencolia I ", ibid., p. 49-67.

15. Drew Daniel, «Between Angel and Dog: Dürer's Melancholy Community », ibid., p. 40-41.

16. Mieke Bal, "Temporal Turbulence », ibid., p. 150151.

17. Voir Georges Didi-Huberman, Le Cube et le visage : autour d'une sculpture d'Alberto Giacometti, Paris, Macula, 1993, p. 159-168.
18. Andrea Bubenik, "The Shape of Things to Come: Dürer's Polyhedron », dans BUBENIK, 2019, p. 68-93.

19. Philippe Despoix, "La Mélancolie et Saturne : un projet collectif au long cours de la bibliothèque Warburg ", dans Revue germanique internationale, $\mathrm{n}^{\circ} 28,2018$, p. 159-181.

20. Karl Giehlow, «Dürers Stich 'Melencolia I' und der maximilianische Humanistenkreis », dans Mitteilungen der Gesellschaft für vervielfältigende Kunst, Vienne, $1903, n^{\circ} 2$, p. $29-41,1904, n^{\circ} 1-2$, p. 6-18, no 4, p. 57-78.

21. Raymond Klibansky, Erwin Panofsky et Fritz Saxl, Saturn and Melancholy: Studies in the History of Natural Philosophy, Religion and Art, Montreal, McGill-Queen's University Press, 2019. De la première édition de 1964, ont paru des traductions italienne en 1983, française en 1989 et allemande en 1992.

22. Erwin Panofsky et Fritz Saxl, Dürers 'Melencolia I'. Eine quellen- und typengeschichtliche Untersuchung, Arpad Weixlgärtner (préface), Leipzig / Berlin, B.G. Teubner ("Studien der Bibliothek Warburg »), 1923.

23. Aby Warburg, "Heidnisch-antike Weissagung in Wort und Bild zu Luthers Zeiten », dans Franz Boll (dir.), Sitzungsberichte der Heidelberger Akademie der Wissenschaften, Heidelberg, Carl Winters Universitäts buchandlung, 1920 ; le texte procède d'une conférence prononcée à Hambourg en 1913 à l'occasion de l'académie d'été de la bibliothèque Warburg (akademische Sommerkurse der kulturwissenschaftlichen Bibliothek Aby Warburg), « Die Planetenbilder auf der Wanderung von Süd nach Nord und ihre Rückkher nach Italien ».

24. Ibid., p. 61 [" humanistisches Trostblatt wider Saturnfürchtigkeit »].

25. Klibansky, Panofsky et Saxl, 1989, cité n. 2, p. 567.

26. Christopher S. Wood, "Senses of Humor », dans Artforum, avril 2020, vol. 58, n 8, p. 34 ("Warburg shut his eyes to the tragic content of the engraving »).

27. MERBACK, 2017, p. 262 (« Dürer's perfectly imperfect image $»$ ).

28. MERBACK, 2017, p. 256 (" astro-medical-philosophical esoterica »).

29. MERBACK, 2017, p. 211 (" vigilant attention to the self $»$ ).

30. C'est pourquoi " l'arpentage », en tant que découpe du monde, peut être qualifié $d^{\prime}$ " art saturnien » (Klibansky, Panofsky et Saxl, 1989, cité n. 2, p. 541) et "l'exacerbation de la conscience de soi » (ibid., p. 371), en tant que mise à distance analytique du sujet, considérée comme inséparable de la mélancolie, comme on le constate par exemple dans les Observations sur la mort et le Jugement dernier de Jacques Legrand, au début du XVe siècle : "À la mesure que la cognoissance vient, le soucy croist, et l'omme se mérancolie plus et plus, selon ce qu'il a de sa condition plus vraie et parfaite cognoissance » (cité ibid., p. 376). Dans ce cadre de pensée, le génie de Dürer est d'avoir institué comme "idée la plus essentielle à [s]a composition [...] l'interpénétration des notions de mélancolie et de géométrie (au sens le plus large) » (ibid., p. 541).

31. Melanie Klein, Deuil et dépression, Paris, Payot \& Rivages, 2003. 
32. D. W. Winnicott, Playing and Reality (1971), cité dans Holly, 2013, p. 64 [" endless memorial search for something in the future that resides in the past »].

33. HOlly, 2013, p. 14, citant Freud, et p. 52, citant Nietzsche [« open wound »].

34. Ibid., p. 116.

35. Ibid., p. XV [« something material beyond all understanding $»]$.

36. Ibid., p. XX et p. 23 [" poetry of loss »].

37. Voir Chari Larsson, "Against a Melancholic Art History. The Afterlife of Images », dans BUBENIK, 2019, p. 193-203.

38. Lettre du 28 décembre 1925, dans Ludwig Binswanger, Aby Warburg, "Correspondance», dans Davide Stimilli (dir.), La Guérison infinie. Histoire clinique d'Aby Warburg (2005), Paris, Payot \& Rivages, 2011, p. 232. Voir Georges Didi-Huberman, «Science avec patience », dans Images Re-vues, hors-série no 4 , 2013 [en ligne, URL : http://journals.openedition.org/ imagesrevues/3024].

39. Ludwig Binswanger, Mélancolie et manie. Études phénoménologiques (1960), Paris, PUF, 1987.

40. Note de Ludwig Binswanger en août 1923 [?], citée dans Joseph Leo Koerner, "Introduction », dans Aby Warburg, Le Rituel du Serpent. Récit d'un voyage en pays pueblo. Art et anthropologie (1923), Paris, Macula, 2003, p. 27. Voir Philippe-Alain Michaud, Aby Warburg et l'image en mouvement, Paris, Macula, 1998, p. 250.

41. Klibansky, Panofsky et Saxl, 1989, cité n. 2, p. 571.

42. Diagnostics du Registre des admissions et du Dr. Emil Kraepelin à la clinique Bellevue de Binswanger à Kreuzlingen, dans Stimilli (dir.), 2007, cité n. 35, p. 14.

43. Jacques Derrida, Spectres de Marx. L'État de la dette, le travail du deuil et la nouvelle Internationale, Paris, Galilée, 1993, p. 31.

44. HOLLY, 2013, p. XIV [" Object's afterlives are as rich and dense as our own »].

45. Didi-Huberman, 2013, cité n. 35. C'est l'auteur qui souligne.

46. HOLLY, 2013, p. 131 [" Lending original works of art an 'as if' animism...»].

47. Frère Laurent du Bois, Somme le roi, ou Livre des vices et des vertus [1279], cité dans Klibansky, Panofsky et Saxl, 1989, cité n. 2, p. 465 (" Accide, cest a dire peresce et anui de bien faire " [acédie, c'est-à-dire paresse et ennui de bien faire »]).

48. VERDIER, 2020, p. 143.

49. Ibid., p. 271.

50. Ibid., p. 328.

51. Francis Picabia, "L'CEil cacodylate", dans Comœedia, 23 novembre 1921, cité ibid., p. 21.

52. Ibid., p. 31.

53. Sur le concept d'estrangement (traduisant l'italien straniamento, traduisant lui-même le russe ostranienie), voir Carlo Ginzburg, " L'estrangement. Préhistoire d'un procédé littéraire ", À distance. Neuf essais sur le point de vue en histoire (1998), Paris, Gallimard, 2001, p. 15-36.
54. Andrea Bubenik, "Introduction", dans BUBENIK, 2019, p. 10 [" Melancholia has been [...] positioned as an archaic humanist condition to be overcome in favour of the eerie »]. Voir Rex Butler, "On Vanishing Land (2013): The Eerie, W. G. Sebald, and English Hauntology ", ibid., p. 94-106.

55. Wendy Brown, "Resisting Left Melancholy », dans Boundary 2, vol. 26, n 3, automne 1999, p. 19-27. Voir Michael Löwy et Robert Sayre, Révolte et mélancolie. Le Romantisme à contre-courant de la modernité, Paris, Payot, 2007 ; et Enzo Traverso, Mélancolie de gauche. La force d'une tradition cachée (XIX'-XXI siècles), Paris La Découverte, 2016.

56. Paul Gilroy, Mélancolie postcoloniale (2004), Paris, B42, 2020, notamment p. 127-128 et p. 148-157. 